\title{
A Meaningful Journey to Onboard Syndromic Data through a Local HIE
}

\author{
Jeffrey Johnson*, Jessica Yen, Brit Colanter and Eric McDonald \\ County of San Diego Public Health Services, San Diego, CA, USA
}

\section{Objective}

This presentation aims to highlight technical approaches, validation activities, outcomes, and lessons learned while onboarding local hospitals through a local health information exchange (HIE) for Meaningful Use Stage 2 syndromic surveillance.

\section{Introduction}

The federal meaningful use initiative is a major driver to the establishment of expanded electronic syndromic surveillance capacity across the United States. Much has been documented about the background and requirements for eligible hospitals to achieve the syndromic meaningful use objectives. However, the role and efforts by public health agencies in the syndromic onboarding process, which varies by jurisdiction, is a significant component of the success of meaningful use.

This presentation will highlight the onboarding process developed by the County of San Diego, Public Health Services, for working with the local hospitals and the local health information exchange. Included will be a review of the technical requirements, project management tools, resources used, challenges encountered, validation techniques, data storage models, the development of performance metrics, and the establishment of protocols for hospital follow-up when detected events using meaningful use. Lastly, a plan to discover the meaning within the syndromic information during the upcoming Stage 2 Year 2 period and the integration of these data into the existing syndromic surveillance system will be described.

\section{Methods}

In the process of onboarding, County of San Diego, Public Health Services, actively engaged with the hospitals, their electronic health record (EHR) vendors, and the local HIE. Public Health Services was actively involved with HL7 message structure validation, connectivity, HL7 message content validation, use case driven testing, and quality assurance. Through these steps, a several lessons have been learned that have led to optimized technical approaches to onboarding future hospitals, as well as gaining insight into how the data can be used in a meaningful way. Going forward, a dedicated meaningful data discovery phase process is being planned for the first 180 days of Stage 2 - Year 2. This will enable insight into the public health value that can be obtained from the meaningful use data. Lastly, the process for integrating the meaningful use syndromic data into the existing syndromic system will be described.

\section{Results}

The local health information exchange in San Diego incorporated as a 501c3 nonprofit in 2013. In 2014, hospitals began to pursue syndromic meaningful use Stage 2 activities. Many hospitals have achieved or are on track to achieve ongoing syndromic data submission capacity by the end of 2014. Several tools, resources, technical approaches, and data management models have been developed as a result of working with the local hospitals. This includes adherence to specification documents, use of the NIST validation tool, and in collaboration with the local HIE. Preliminary outcomes have shown that it is valuable to have public health involved in the onboarding process and, more importantly, during validation activities. A significant challenge identified is that many hospitals have different information workflows that manifest outwardly in the submitted data. These include differing classification of inpatient and ambulatory related messages, message segment values and how the patient's treating location is coded.

\section{Conclusions}

Public health agencies across the United States vary in their capacity to onboard meaningful use syndromic data. At the same time, various technology approaches are currently in place or are in development to collaborate with the hospitals pursuit of Stage 2 - Year 2 attestation. Following a collaborative onboarding process with the public health agency as a key stakeholder is critical to understanding and using meaningful use syndromic information.

\section{Keywords}

meaningful use; syndromic surveillance; onboarding; health information exchange; validation

*Jeffrey Johnson
E-mail: Jeffrey.Johnson@sdcounty.ca.gov 